\title{
Avoiding Unnecessary Exposure to General Anesthesia for Dental Treatments in the First Years of Life
}

\author{
Nahid Ramazani (iD) ${ }^{1,}$ \\ ${ }^{1}$ Department of Pediatric Dentistry, Children's and Adolescents' Health Research Center, Oral and Dental Disease Research Center, Zahedan University of Medical Sciences, \\ Zahedan, Iran \\ 'Corresponding author: Department of Pediatric Dentistry, Children's and Adolescents' Health Research Center, Oral and Dental Disease Research Center, Zahedan University \\ of Medical Sciences, Zahedan, Iran. Email: ramazani77@gmail.com
}

Received 2020 August 20; Revised 2020 December 06; Accepted 2020 December 17.

Keywords: Dental Treatment, General Anesthesia, Pediatric Dentistry

\section{Dear editor,}

I read the article entitled "Risk of attention deficit hyperactivity disorder after early exposure to general anesthesia; A case control study", by Sedighnejad et al. (1), published in the third issue of the Iranian Journal of Pediatrics (2020) with great interest. The researchers concluded that early general anesthesia (GA) could be a predisposing factor for the subsequent development of attention deficit hyperactivity disorder(ADHD). They found that a history of GA in children younger than four years of age was a significant predictor of ADHD (1). After reading this article, as a pedodontist, I was concerned about the longterm anesthetic-induced neurodevelopmental outcomes of common dental procedures under GA. Therefore, I was encouraged to conduct further research on this subject.

It is widely accepted that GA is a suitable tool for surgery and invasive diagnostic procedures requiring immobilization $(2,3)$. Generally, GA is an unavoidable technique, especially in children due to their age-specific characteristics and limited cooperation (2). In pediatric dentistry, dental treatment under GA has been integrated into clinical practice for more than a few decades (4). Generally, children who fail to cope with routine in-office dental procedures receive treatment under GA (5). The majority of these cases are healthy children with early childhood caries, for whom common behavioral management techniques are ineffective and infeasible (6). All dental treatments, including pulp therapy, restoration, and extraction, can be performed in one single session by using GA.

Generally, the advantages of pediatric GA, especially pain relief, anxiety reduction, and ideal surgical performance, which are prerequisites for an appropriate treat- ment or diagnostic intervention, are well-established (1, $3)$. If experienced professionals adhere to scientific guidelines, treatments can be safely performed (7). Evidence shows that the mortality rate is less than 1 per 3.5 million dental GA cases (7). However, some risks and disadvantages revealed over time are attributed to GA performed in early life (8). There are some concerns about the GA safety, most of which are related to neurological toxicity in young susceptible brains (2). In this regard, previous studies have reported neurotoxicity induced by GA in immature animals (1-3). Generally, there are controversies about the safety of GA for the pediatric population $(1,3)$; however, it is difficult to confirm the significance of these findings in clinical practice (1). Also, considering the adverse effects of GA on the development of the central nervous system, it is difficult to determine the exact vulnerable age range and exposure time (1). Nonetheless, the majority of previous studies have reported the importance of the number, duration, and timing of exposure to GA, irrespective of the type of anesthetic agent and route of administration $(1,2,9)$.

The brain of individuals may be affected by GA, particularly up to the age of 2 - 3 years $(2,3)$; this is especially true for treatments that continue for more than three hours, including dental procedures (9). It is worth mentioning that dental treatments for children under GA takes an average of 1 - 4 hours, depending on the conditions (10).

Also, over time and with age, developing nervous system still continues to be sensitive but less than first 2 years of life (3). According to one previous study, age below four years can be defined as the risky period for GA (1); overall, the critical time is the period of peak synaptogenesis (2). Anesthetic exposure may cause long-lasting apoptotic degeneration of the nervous system (1-3). There are known 
and unknown confounders that affect the relationship between GA modality and the nervous system development (1). However, early and unnecessary GA exposure must be generally avoided under the age of three years (2).

So far, the main cause of delayed brain development due to GA has not been clarified, and further studies are required to provide more information about the neurological outcomes of GA. For further assessments, neurodevelopmental monitoring of individuals with a history of anesthesia exposure may reveal valuable evidence about neurological impairment. In certain cases, the only practical option for pediatric dental treatments is GA; nevertheless, anesthesia should be considered as the last resort due to its potential risks. Until the outcomes of pediatric dental practices under GA are discovered, it is important to weigh the advantages and disadvantages of these procedures. Another way to reduce the complications is to postpone dental GA as long as it does not cause any dental pain or tooth loss in patients. Besides avoiding repeated dental GA, it is recommended that at the same time of considering the urgency of the treatment, to wait until first dentition is fully erupted. Nevertheless, unnecessary GA exposure must be always avoided.

\section{Footnotes}

Authors' Contribution: NR evaluated related articles, extracted useful content from them and drafted the letter, revised and approved it.

Conflict of Interests: There is no conflict of interest. Funding/Support: There is no funding.

\section{References}

1. Sedighnejad A, Soltanipour S, Saberi A, Kousha M, Bidabadi E, Biazar G, et al. Risk of Attention Deficit Hyper Activity Disorder After Early
Exposure to General Anesthesia; A Case Control Study. Iran J Pediatrics. 2020;30(3). doi: 10.5812/ijp.99976.

2. Walkden GJ, Pickering AE, Gill H. Assessing Long-term Neurodevelopmental Outcome Following General Anesthesia in Early Childhood: Challenges and Opportunities. Anesth Analg. 2019;128(4):68194. doi: 10.1213/ANE.0000000000004052. [PubMed: 30883414]. [PubMed Central: PMC6436726].

3. Lee JH, Zhang J, Wei L, Yu SP. Neurodevelopmental implications of the general anesthesia in neonate and infants. Exp Neurol. 2015;272:50-60. doi: 10.1016/j.expneurol.2015.03.028. [PubMed: 25862287]. [PubMed Central: PMC4598257].

4. Lee PY, Chou MY, Chen YL, Chen LP, Wang CJ, Huang WH. Comprehensive dental treatment under general anesthesia in healthy and disabled children. Chang Gung Med J. 2009;32(6):636-42. [PubMed: 20035643].

5. Tsai CL, Tsai YL, Lin YT, Lin YT. A retrospective study of dental treatment under general anesthesia of children with or without a chronic illness and/or a disability. Chang Gung MedJ.2006;29(4):412-8. [PubMed: 17051840].

6. Baghdadi ZD. Children's oral health-related quality of life and associated factors: Mid-term changes after dental treatment under general anesthesia. J Clin Exp Dent. 2015;7(1):e106-13. doi: 10.4317/jced.51906. [PubMed: 25810820]. [PubMed Central: PMC4367996].

7. Roberts GJ, Mokhtar SM, Lucas VS, Mason C. Deaths associated with GA for dentistry 1948 - 2016: the evolution of a policy for general anaesthesia (GA) for dental treatment. Heliyon. 2020;6(1). e02671. doi: 10.1016/j.heliyon.2019.e02671. [PubMed: 31938737]. [PubMed Central: PMC6953638].

8. Farsi N, Ba'akdah R, Boker A, Almushayt A. Postoperative complications of pediatric dental general anesthesia procedure provided in Jeddah hospitals, Saudi Arabia. BMC Oral Health. 2009;9:6. doi: 10.1186/1472-6831-9-6. [PubMed: 19228406]. [PubMed Central: PMC2667174].

9. Bartels DD, McCann ME, Davidson AJ, Polaner DM, Whitlock EL, Bateman BT. Estimating pediatric general anesthesia exposure: Quantifying duration and risk. Paediatr Anaesth. 2018;28(6):5207. doi: 10.1111/pan.13391. [PubMed: 29722100]. [PubMed Central: PMC6291204].

10. VanCleave AM, Jones JE, McGlothlin JD, Saxen MA, Sanders BJ, Walker LA. Factors involved in dental surgery fires: a review of the literature. Anesth Prog. 2014;61(1):21-5. doi: 10.2344/0003-3006-61.1.21. [PubMed: 24697822]. [PubMed Central: PMC3975610]. 